\title{
Jianpi Yiqi Shexue ameliorates immune thrombocytopenia related fatigue by regulating mitochondrial function
}

\author{
Yayue Zhang ${ }^{1}$, Jia Wang', Jieyin Gao ${ }^{3}$, Ming Guo ${ }^{3}$, Haiyan Chen ${ }^{3}$ Junxia Liu ${ }^{3}$,ing Li $^{3}$, Junyao Liao ${ }^{3}$, \\ Xinyi Chen ${ }^{1}$, Haiyan Lang ${ }^{3}$ \\ ${ }^{1}$ Department of Oncology and Hematology, Dongzhimen Hospital Affiliated to Beijing University of Chinese Medicine, Beijing, China; ${ }^{2}$ Department \\ of Integrated Traditional and Western Medicine, Affiliated Cancer Hospital of Zhengzhou University, Henan Cancer Hospital, Zhengzhou, China; \\ ${ }^{3}$ Department of Hematology, Dongfang Hospital Affiliated to Beijing University of Chinese Medicine, Beijing, China \\ Contributions: (I) Conception and design: Y Zhang, X Chen, H Lang; (II) Administrative support: X Chen, H Lang; (III) Provision of study \\ materials or patients: J Wang, J Gao; (IV) Collection and assembly of data: M Guo, J Liu; (V) Data analysis and interpretation: Y Zhang, J Gao; (VI) \\ Manuscript writing: All authors; (VII) Final approval of manuscript: All authors. \\ Correspondence to: Haiyan Lang. Department of Hematology, Dongfang Hospital Affiliated to Beijing University of Chinese Medicine, 6 \\ Fangxingyuan 1st Block, Fengtai District, Beijing 100078, China. Email: julietlang@126.com.
}

Backgroundk Immune thrombocytopenia (ITP) is a heterogeneous autoimmune disease characterized by platelet destruction. In previous studies, Jianpi Yiqi Shexue (JPYQSX) was shown to increase the peripheral platelet (PLT) counts in patients with ITP. In addition, JPYQSX also dramatically alleviated weakness and fatigue. This study aimed to investigate the effect of JPYQSX on ITP related fatigue and to illuminate the underlying mechanisms of its therapeutic effects.

Methods: Prednisone and different doses of JPYQSX were orally administered to mice with ITP. Posttreatment, all mice were subjected to a forced swimming test. In addition, blood samples were analyzed using an automated hematology analyzer. Spleen, liver, lungs, heart, kidneys, and colon tissues were collected to determine the expressions of reactive oxygen species (ROS), adenosine triphosphate (ATP), mitochondrial DNA (mtDNA), succinate dehydrogenase complex flavoprotein subunit A (SDHA), caseinolytic mitochondrial matrix peptidase proteolytic subunit (ClpP), and Lon peptidase 1 (Lonp1).

Results: Compared with the vehicle group, JPYQSX prolonged the forced swimming time, increased the PLT counts, and reduced the liver and spleen indices \{calculated as follows: organ index (\%) = [organ weight $(\mathrm{mg})$ /body weight $(\mathrm{g})] \times 100 \%$ \}. In addition, the levels of ROS were upregulated, while the ATP and mtDNA contents were downregulated in ITP model mice. Administration of JPYQSX restored the expression of these mitochondrial molecules to normal levels. Furthermore, JPYQSX also decreased the expressions of SDHA, ClpP, and Lonp1, which are closely related to mitochondrial activity.

Conclusions: These findings suggest that JPYQSX prevents antiplatelet sera-mediated platelet destruction in ITP mice and ameliorates fatigue possibly through its effect on mitochondrial function. This study revealed JPYQSX as a potential alternative approach for ITP therapy.

Keywords: Jianpi Yiqi Shexue; immune thrombocytopenia (ITP); fatigue; mitochondrial function; succinate dehydrogenase flavoprotein subunit

Submitted Sep 20, 2020. Accepted for publication Dec 01, 2020.

doi: 10.21037/apm-20-2153

View this article at: http://dx.doi.org/10.21037/apm-20-2153

(c) Annals of Palliative Medicine. All rights reserved. 


\section{Introduction}

Immune thrombocytopenia (ITP) is an acquired hematological disease characterized by platelet destruction and megakaryocyte dysfunction (1). Patients typically present with variable severity of bleeding, especially in the skin and the mucosa (2). The estimated prevalence of ITP is 10/100,000 adults; the estimated annual incidence rate ranges from 1.6-3.9/100,000 persons (3). Individuals of all ages may be affected by ITP, though the condition is more acute in children than in adults. However, the disease is selflimiting in the vast majority of children. In contrast, ITP in adults is more often a chronic disorder that clinically presents with weakness, myalgia, arthralgia, shortness of breath, and purpura. Apart from the risks associated with bleeding, the accompanying symptoms, such as fatigue, have a detrimental effect on the daily lives of patients.

A rare autoimmune disease, various immune cells (such as T cells, B cells, macrophages, dendritic cells, and mesenchymal stem cells) have been implicated in the pathogenesis of ITP (4). Corticosteroids have traditionally been used as the standard first-line therapy for ITP; intravenous immunoglobulin and anti-D immunoglobulin are generally applied as emergency rescue therapies that have a transient response (5). Several novel therapies and immunosuppressive medications have changed the management landscape of ITP. In recent years, several drugs such as rituximab and thrombopoietin receptor agonists (TPO-RA) have been increasingly used to postpone splenectomy; the reported long-term response rate of these therapies is $66 \%(6,7)$. However, the current treatments are mainly focused on extending the lifespan and reducing the mortality of patients with ITP. The development of therapeutic regimens that improve the quality of life of these patients is a key imperative.

Traditional Chinese medicines (TCMs) are complementary and alternative medicines that have been successfully used in the treatment of immune-related diseases (8), and confer several therapeutic advantages over Western medical treatment. They have been shown to enhance immune function and alleviate immune deficiencyrelated symptoms. In TCM, ITP has been referred to as "purpura disease", and differentiated as "spleen deficiency". Jianpi Yiqi Shexue (JPYQSX) is a Chinese formula developed based on the TCM syndrome analysis of ITP. The JPYQSX formula includes herbs such as Astragalus membranaceus (Huangqi), Codonopsis pilosula (Dangshen), Atractylodes macrocephala Koidz (Baizhu), and Poria cocos (Fuling). It is designed to invigorate the spleen and supplement qi, which ultimately reduces the frequency and severity of bleeding. In a clinical study, JPYQSX administered in combination with recombinant human thrombopoietin (RHTPO) was shown to improve the quality of life of patients; based on TCM syndrome score analysis, the study documented alleviation of fatigue, ventosity, and poor appetite (9). Studies have shown that increased oxidative phosphorylation (OXPHOS), as reflected by increased mitochondrial activity, along with impaired mitochondrial stress response, can severely compromise the regeneration of hematopoietic stem cells (HSCs) (10). Moreover, mitochondrial stress is closely related to spleen deficiency. Therefore, in this study, we tested the hypothesis that JPQYSX protects against platelet destruction and ameliorates the fatigue of patients with ITP by regulating mitochondrial function.

We present the following article in accordance with the ARRIVE reporting checklist (available at http://dx.doi. org/10.21037/apm-20-2153).

\section{Methods}

\section{Herbs and reagents}

Lyophilized enzyme-linked protein A was purchased from Boshide Biological Technology Co. Ltd. (Wuhan, Hubei, China). Gelatin, Tween-20 was obtained from Kehao Biological Technology Co. Ltd. (Xi'an, Shaanxi, China). Chloral hydrate was provided by Sinopharm Chemical Reagent Co., Ltd. (Shanghai, China). Paraformaldehyde was obtained from Solarbio Life Sciences Co. Ltd. (Beijing, China). Disodium 4-nitrophenylphosphate and diethanol amine were provided by the Tianjin Kemiou Chemical Reagent Co. Ltd. (Jinnan, Tianjin, China). Mannitol and dimethyl sulfoxide (DMSO) were purchased from Sigma-Aldrich (St. Louis, MO, USA). Prednisone was purchased from Jingchun Biochemical Technology Co. Ltd. (Shanghai, China).

The extract of JPYQSX, containing Astragalus membranaceus (Huangqi, $20 \mathrm{~g}$ ), Codonopsis pilosula (Dangshen, $15 \mathrm{~g}$ ), Poria cocos (Fuling, $15 \mathrm{~g}$ ), Atractylodes macrocephala Koidz (Baizhu, $10 \mathrm{~g}$ ), Equus asinus L. (Ejiao, $15 \mathrm{~g}$ ), Rubia cordifolia L. (Qiancao, $10 \mathrm{~g}$ ), Glycyrrbiza uralensis Fisch. (Zhigancao, $6 \mathrm{~g}$ ), was prepared by the Xinghua Institute of Pharmacology (Taiyuan, Shanxi, China), according to technological standards for the preparation of TCMs.

\section{Animals}

All experiments were conducted in compliance with the 
Table 1 Animal grouping and treatment

\begin{tabular}{lcccc}
\hline Group & $\mathrm{n}$ & Treatment & Modeling time $(\mathrm{d})$ & Administration time $(\mathrm{d})$ \\
\hline Control & 18 & $0.9 \%$ normal saline & 8 & 8 \\
Vehicle & 18 & $0.9 \%$ normal saline & 8 & 8 \\
Prednisone & 18 & Prednisone solution & 8 & 8 \\
JPYQSX (low dose) & 18 & JPYQSX prescription & 8 & 8 \\
JPYQSX (high dose) & 18 & JPYQSX prescription & 8 & 8 \\
\hline
\end{tabular}

All mice were orally administrated at $0.1 \mathrm{~mL} / 10 \mathrm{~g}$ body weight. Prednisone solution was prepared at a concentration of $2 \mathrm{mg} / \mathrm{mL}$. JPYQSX prescription was prepared at a concentration of $410.0 \mathrm{~g} / \mathrm{L}$ for low dose group, and $820.0 \mathrm{~g} / \mathrm{L}$ for high dose group. JPYQSX, Jianpi Yiqi Shexue; d, days.

Guidelines for the Care and Use of Laboratory Animals. Experimental protocols were approved by the Committee of Ethics of Animal Experimentation of the Air Force Medical University (No. XYLS2019069). A total of 90 nonpregnant $\mathrm{BALB} / \mathrm{c}$ mice (aged 8 weeks; weight 18-22 g; 45 male and 45 females) were purchased from the Experimental Animal Center of the Air Force Medical University (Code: SCXKShan 2014-002). A total of 24 guinea pigs (weight $250 \mathrm{~g}$; 12 male and 12 female) were purchased from the Xingping Tianrui Laboratory Animal Center (Code: SCXK-Shan 2012-001). Animals were housed in a breeding room for 1 week prior to the experiments under controlled environmental conditions (constant humidity and temperature). Mice were fasted overnight and an additional $12 \mathrm{~h}$ prior to the administration of drugs.

\section{ITP model and grouping}

The detailed procedure for the establishment of the ITP mouse model is described elsewhere $(11,12)$. We first prepared anti-platelet sera; in brief, thirty BALB/c mice were euthanized and whole blood samples were collected by cardiac puncture in ethylenediaminetetraacetic acid (EDTA)-pretreated tubes. The blood samples were centrifuged at 1,000 rpm for $10 \mathrm{~min}$. The supernatant containing abundant platelets was collected and recentrifuged at 3,000 $\mathrm{rpm}$ for another $10 \mathrm{~min}$. The precipitates were collected, washed twice with phosphate buffered saline (PBS), and then resuspended and diluted to obtain a final concentration of $2.5 \times 10^{6} / \mathrm{mL}$. The harvested platelets were mixed with adjuvants in a ratio of 1:1 and used as antigens. The 24 guinea pigs were initially immunized by a $1 \mathrm{~mL}$ subcutaneous injection of a mixture of platelets and Freund's complete adjuvants in the footpads and back (5 injection sites in total). The second, third, and fourth immunizations were administered at weeks 1, 2, and 4, respectively, with a mixture of platelets and Freund's incomplete adjuvants at the same sites. Whole blood was withdrawn by cardiac puncture in the fifth week. The blood was centrifuged at $1,000 \mathrm{r} / \mathrm{min}$ for $10 \mathrm{~min}$. The sera were collected and inactivated at $56^{\circ} \mathrm{C}$ for $30 \mathrm{~min}$, heapsorted twice, and diluted with normal saline (NS) in a ratio of 1:4. The antiplatelet sera (APS) were tittered as described in the literature. The titer of APS in the immunized guinea pigs was 2 -fold higher than that in the naïve guinea pigs.

After determining the platelet counts in peripheral blood using an automated hematology analyzer (PERLONG XFA6130, China), $90 \mathrm{BALB} / \mathrm{c}$ mice were randomly allocated into a control group, vehicle group, prednisone group, low-dose JPYQSX group, and high-dose JPYQSX group. With the exception of the control group, mice in the other 4 groups received an injection of APS $(100 \mu \mathrm{L} / 20 \mathrm{~g}$ body weight) every 2 days for a total of 4 times. Mice in the control group received an injection of NS instead. On day 9 , all mice were initiated on the following oral treatment at a dose of $0.1 \mathrm{~mL} / 10 \mathrm{~g}$ body weight: the control and vehicle mice received $0.9 \% \mathrm{NS}$, prednisone group received prednisone solution $(2 \mathrm{mg} / \mathrm{mL})$, and JPYQSX groups received JPYQSX (410.0 g/L for low dose, $820.0 \mathrm{~g} / \mathrm{L}$ for high dose). This treatment regimen was administered for 8 days. The schedule is presented in Table 1 .

\section{Forced swimming test and sample collection}

The forced swimming test was carried out to evaluate the fatigue of mice according to previous studies. All mice were subjected to forced swimming with loads constituting $10 \%$ body weight at 8 days post-treatment. The swimming time was recorded and compared between the groups. Blood parameters were measured to assess efficacy of the drugs. 
Table 2 Primers of SDHA, LonP1, ClpP, and GAPDH

\begin{tabular}{llc}
\hline mRNA & Direction & Sequences \\
\hline SDHA & Forward & 5'-CCATAGTGGTGGAGCAGACG-3' \\
& Reverse & 5'-AGAACAACAGCTGGGCAATG-3' \\
LonP1 & Forward & 5'-AGAACTTGGACTTGGCTCGG-3' \\
& Reverse & 5'-AGCTGGCTGACTGCAATGAA-3' \\
ClpP & Forward & 5'-CCATAGTGGTGGAGCAGACG-3' \\
& Reverse & 5'-AGAACAACAGCTGGGCAATG-3' \\
GAPDH & Forward & 5'-GTCATCCCAGAGCTGAACGG-3' \\
& Reverse & 5'-TACTTGGCAGGTTCTCCAGG-3' \\
\hline
\end{tabular}

SDHA, succinate dehydrogenase complex flavoprotein subunit A; ClpP, caseinolytic mitochondrial matrix peptidase proteolytic subunit; LonP1, Lon peptidase 1; GAPDH, glyceraldehyde 3-phosphate dehydrogenase.

Briefly, $10 \mu \mathrm{L}$ of serum samples of mice were collected from the tail vein prior to the experiment and at 2, 4, and 8 days after APS injection, and at 4 and 8 days after the completion of treatment. The levels of platelet distribution width (PDW), hemoglobin (HGB), and white blood cell (WBC) counts were determined using the automatic hematology analyzer. The weight of mice was recorded prior to and immediately after the experiment. At the endpoint, all mice were anesthetized and sacrificed by cervical dislocation. The spleen, liver, heart, lung, kidney, colon, and brain were extracted and weighed. The organ index was calculated as follows: organ index $(\%)=$ [organ weight (mg)/body weight (g)] $\times 100 \%$.

\section{Measurement of mitochondrial function}

Mitochondrial DNA (mtDNA), reactive oxygen species (ROS), and adenosine triphosphate (ATP) were determined to evaluate mitochondrial function. Fresh spleens were isolated from mice and immediately frozen using liquid nitrogen; subsequently, $4 \mathrm{~mm}$ thick slices were prepared. A total ROS assay kit (Thermo Fisher, Waltham, MA, USA) was used to detect the ROS content in the spleen. Qualitative fluorescence analysis was conducted using a fluorescence microscope (OLYMPUS, Japan). The intensity of red fluorescence was positively associated with ROS concentration. The total DNA was extracted from spleen tissue using the PicoPure $^{\mathrm{TM}}$ DNA Extraction Kit (Thermo Fisher, Waltham, MA, USA), and amplified and analyzed by real-time fluorescent quantitative polymerase chain reaction (qPCR). The ATP content was measured using an ATP determination kit (Thermo Fisher,
Waltham, MA, USA). The relative light unit (RLU) was determined using chemiluminescence apparatus. The amount of ATP in the colon tissue was calculated according to the standard curve of RLU.

\section{Reverse transcription-polymerase chain reaction}

Total RNA was isolated from tissues using TRIzol reagent (Thermo Fisher Scientific, Waltham, MA, USA) according to the manufacturer's instruction; it was used to generate singlestranded cDNA for qRT-PCR. The glyceraldehyde 3-phosphate dehydrogenase (GAPDH) gene was used as an internal control. Relative mRNA expression levels were normalized to those of GAPDH and calculated according to the $2^{-\Delta \Delta C T}$ method. The primers used are presented in Table 2.

\section{Western blot analysis}

Proteins were isolated from the spleen, liver, lungs, kidneys, heart, and colon. Equal amounts of protein $(10 \mu \mathrm{g})$ were subjected to the wells for sodium dodecyl polyacrylamide gel electrophoresis (SDS-PAGE) separation, followed by their transfer on to polyvinylidene fluoride (PVDF) membranes. Subsequently, the membranes were incubated overnight with the primary antibodies at $4{ }^{\circ} \mathrm{C}$, followed by incubation with horseradish peroxidase (HRP)-conjugated secondary antibodies for $2 \mathrm{~h}$. The following primary antibodies were used: anti-succinate dehydrogenase complex flavoprotein subunit A (SDHA) (1:2,000, Abcam, Cambridge, MA, USA), anti-caseinolytic mitochondrial matrix peptidase proteolytic subunit (ClpP) (1:1,000, Abcam, Cambridge, MA, USA), anti-Lon peptidase 1 (LonP1) (1:1,000, Abcam, Cambridge, MA, USA), and anti-GAPDH (1:10,000, Abcam, USA).

\section{Statistical analyses}

Continuous variables are presented as mean \pm standard deviation (SD) and between-group differences were assessed using analysis of variance (ANOVA) and t-test. All statistical analyses were performed using the SPSS version 24.0 (IBM, Armonk, NY, USA) software; P values $<0.05$ were considered indicative of statistical significance.

\section{Results}

\section{Forced swimming test}

There were no significant between-group differences with 
Table 3 Forced swimming test of BALB/c mice in different groups (time/s)

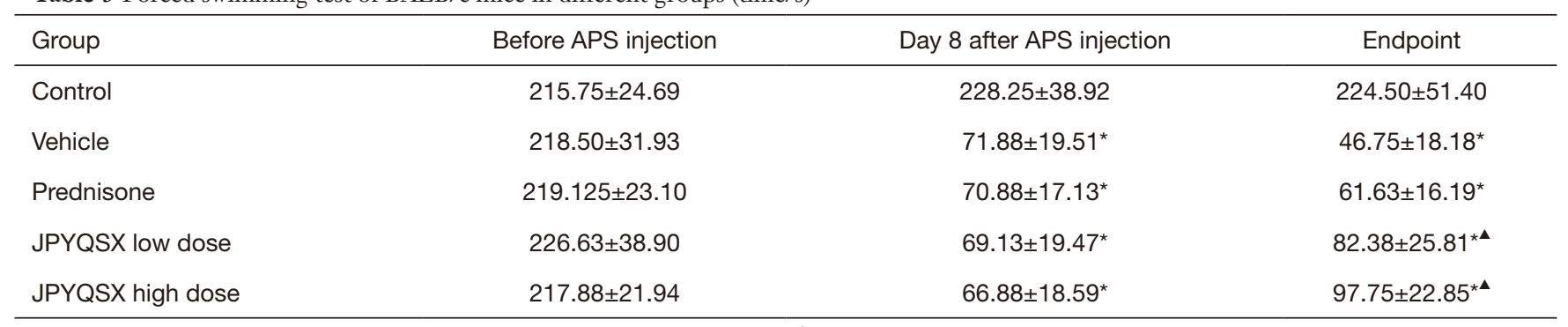

Compared with control group, ${ }^{*} \mathrm{P}<0.05$; compared with vehicle group, ${ }^{\wedge} \mathrm{P}<0.01$. APS, antiplatelet serum; JPYQSX, Jianpi Yiqi Shexue.

Table 4 Organ index in ITP modeling mice after treatment

\begin{tabular}{|c|c|c|c|c|c|}
\hline Parameters & Control & Vehicle & Prednisone & JPYQSX low dose & JPYQSX high dose \\
\hline Spleen index $(\mathrm{mg} / \mathrm{g})$ & $0.42 \pm 0.20$ & $1.30 \pm 0.37^{* *}$ & $0.72 \pm 0.20^{\star \star \Delta}$ & $1.04 \pm 0.29^{\star \star \Delta}$ & $1.06 \pm 0.27^{\star \star \Delta}$ \\
\hline Liver index (mg/g) & $4.58 \pm 0.38$ & $5.13 \pm 0.33^{\star \star}$ & $5.10 \pm 0.38^{\star \star}$ & $4.71 \pm 0.62^{\Delta}$ & $4.61 \pm 0.53^{\wedge}$ \\
\hline Heart index (mg/g) & $0.55 \pm 0.05$ & $0.58 \pm 0.07^{\star}$ & $0.63 \pm 0.10^{*}$ & $0.63 \pm 0.13^{*}$ & $0.58 \pm 0.08$ \\
\hline Kidney index (mg/g) & $1.59 \pm 0.22$ & $1.60 \pm 0.25$ & $1.50 \pm 0.24$ & $1.61 \pm 0.26$ & $1.63 \pm 0.17$ \\
\hline Colon index (mg/g) & $0.76 \pm 0.18$ & $0.86 \pm 0.23$ & $0.82 \pm 0.31$ & $0.94 \pm 0.29^{*}$ & $0.83 \pm 0.21$ \\
\hline Brain index (mg/g) & $1.66 \pm 0.20$ & $1.82 \pm 0.21^{*}$ & $1.70 \pm 0.37$ & $1.69 \pm 0.31$ & $1.80 \pm 0.39$ \\
\hline
\end{tabular}

Compared with control group, ${ }^{*} \mathrm{P}<0.05$, ${ }^{* *} \mathrm{P}<0.01$; compared with vehicle group, ${ }^{\Delta} \mathrm{P}<0.05,{ }^{\wedge} \mathrm{P}<0.01$. JPYQSX, Jianpi Yiqi Shexue.

respect to forced swimming time prior to the experiment (Table 3). However, all experiment groups showed a marked decrease in forced swimming time on day 8 after APS injection and at the endpoint of the experiments, as compared to that in the control group $(\mathrm{P}<0.01)$. Of note, the forced swimming time in the JPQYSX groups were significantly longer than that of the vehicle group and the prednisone group $(\mathrm{P}<0.01)$; however, there were no significant differences between the two JPQYSX groups in this respect $(\mathrm{P}>0.05)$.

\section{Influence of $\mathcal{P P Q Y S X}$ on the organ index}

To investigate the effects of drugs on organ index, prednisone, low-dose JPQYSX, and high-dose JPQYSX were orally administered to APS-induced mice for 8 consecutive days. As shown in Table 4, there were no significant betweengroup differences with respect to body weight. At the end of the experiment, the spleen, liver, heart, and brain indices in the experiment groups were significantly greater than that in the control group $(\mathrm{P}<0.01, \mathrm{P}<0.01, \mathrm{P}<0.05$, and $\mathrm{P}<0.05$, respectively); however, there no significant between-group differences with respect to kidney index $(\mathrm{P}>0.05)$. When compared to the vehicle group, the prednisone group showed a decreased spleen index, and the JPQYSX group showed a decreased liver index. The lung and colon indices were significantly higher in the prednisone group $(\mathrm{P}<0.05)$ and in the low-dose JPQYSX group, respectively.

\section{Effects of $\mathcal{F P Q Y S X ~ o n ~ p e r i p h e r a l ~ b l o o d ~ p i c t u r e ~}$}

There was no significant difference in terms of platelet (PLT) counts among the 5 groups before the experiment (Table 5, P>0.05). However, PLT levels were markedly decreased at days 4 and 8 after APS injection $(\mathrm{P}<0.05)$. Following APS injection, prednisone, low-dose JPQYSX, and high-dose JPQYSX were orally administered to APSinduced mice for 8 consecutive days; the PLT level in the vehicle group was still lower than that in the control group $(\mathrm{P}<0.01)$. However, the high-dose JPQYSX group showed a higher PLT level compared to the vehicle group at day 4 $(\mathrm{P}<0.01)$ and the prednisone and low-dose JPQYSX groups showed higher PLT levels compared to the vehicle group 
Table 5 Peripheral platelet counts at different time points

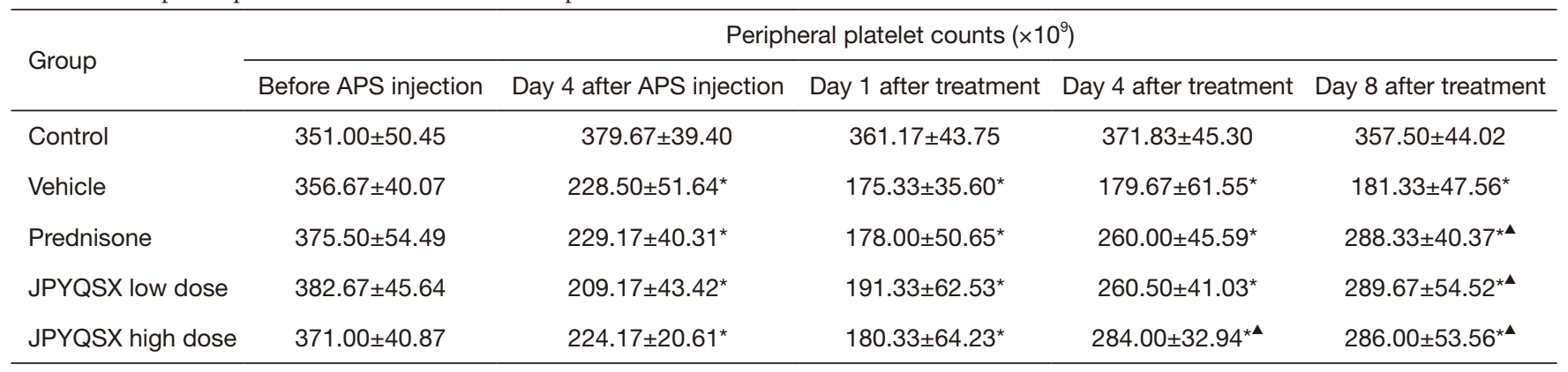

Compared with control group, ${ }^{*} \mathrm{P}<0.05$. Compared with vehicle group, ${ }^{\wedge} \mathrm{P}<0.01$. JPYQSX, Jianpi Yiqi Shexue; APS, antiplatelet serum.

Table 6 Blood picture of mice in different groups at different time points

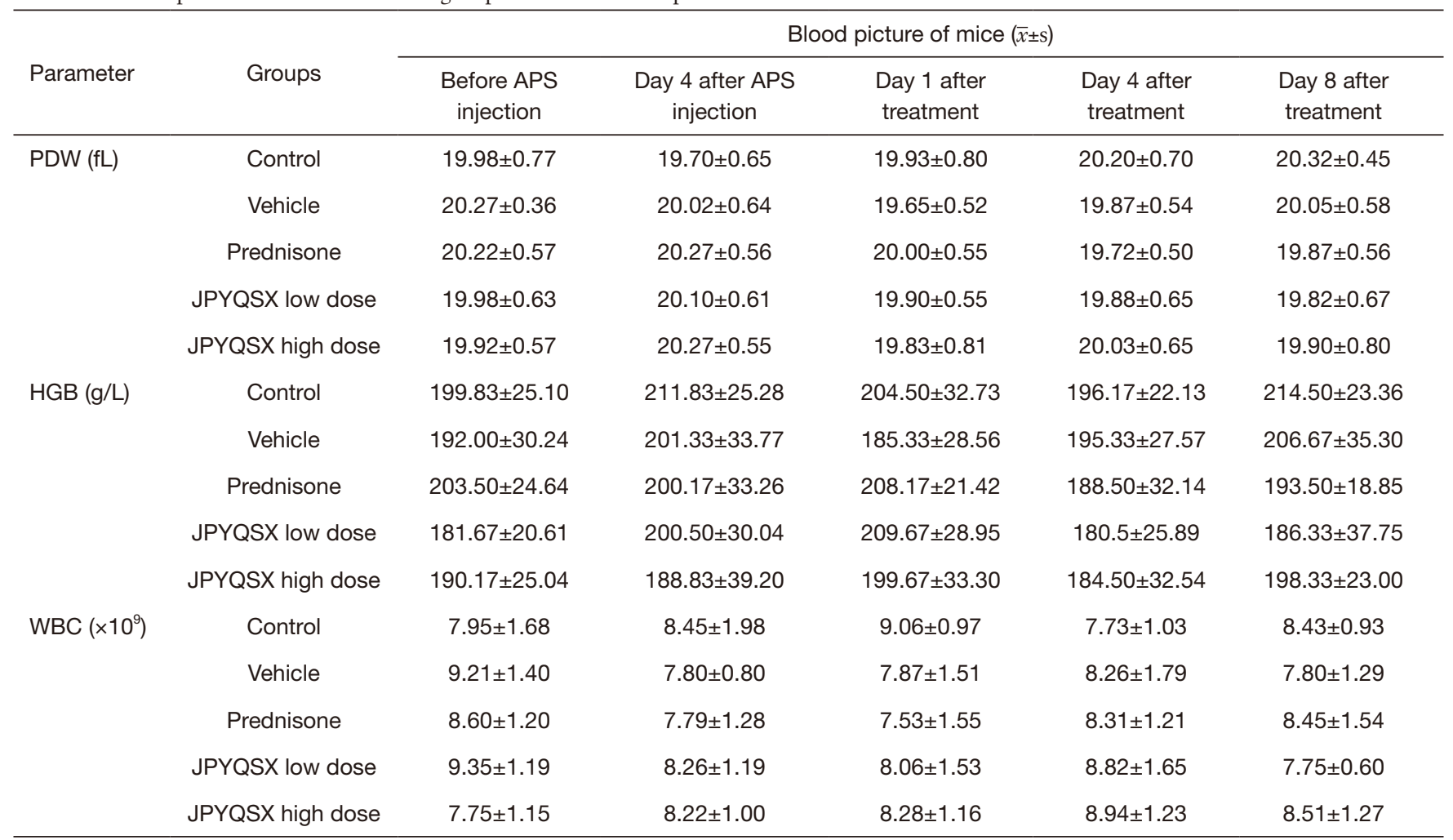

JPYQSX, Jianpi Yiqi Shexue; PDW, platelet distribution width; HGB, hemaglobin; WBC, white blood cell.

until day $8(\mathrm{P}<0.01)$. No significant difference was observed among the 5 groups at any time-point in terms of PDW, HGB, or WBC counts (Table 6, $\mathrm{P}>0.05$ ).

\section{Impact of $\mathcal{F P Y Q S X ~ o n ~ m i t o c h o n d r i a l ~ f u n c t i o n ~}$}

The levels of ROS were significantly increased in mice treated with a vehicle compared to the control group
(Figure 1). However, the ROS levels were markedly decreased after the administration of prednisone, low-dose JPQYSX, and high-dose JPQYSX. However, the ROS level in the high-dose JPYQSX group was higher than that in the prednisone group $(\mathrm{P}<0.01)$, and lower than that in the low-dose JPQYSX group after administration of drugs $(\mathrm{P}<0.05)$. The levels of mtDNA in the experiment groups were significantly decreased as compared to that 

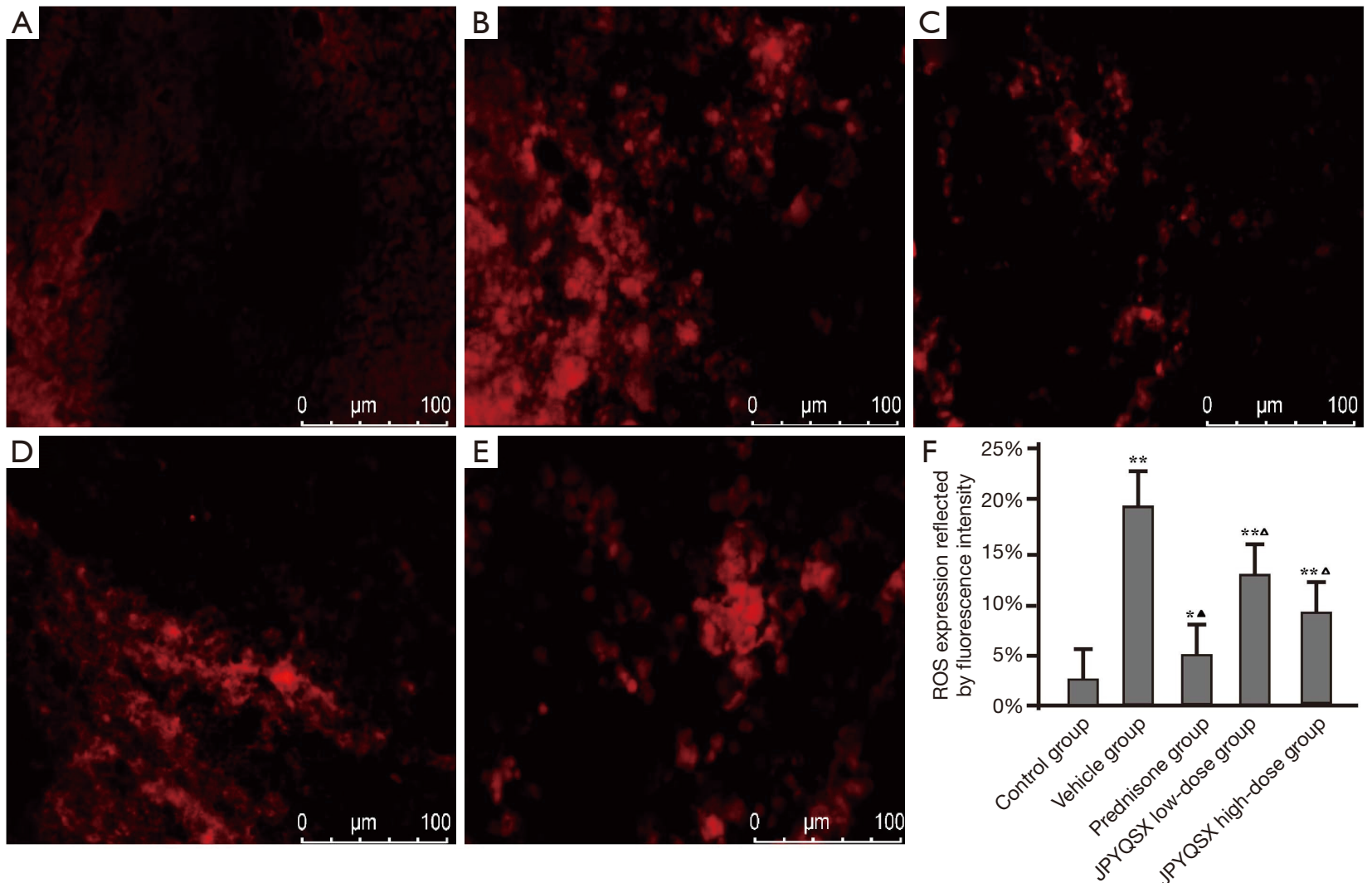

Figure 1 ROS expression in spleen tissues reflected by fluorescence intensity. (A) Control group; (B) vehicle group; (C) prednisone group; (D) low-dose JPYQSX group; (E) high-dose JPYQSX group; (F) bar graph showing the ROS expression as reflected by fluorescence intensity. ${ }^{*} \mathrm{P}<0.05$, ${ }^{* *} \mathrm{P}<0.01$ versus control group; ${ }^{\wedge} \mathrm{P}<0.05$ versus vehicle group; ${ }^{\wedge} \mathrm{P}<0.01$ versus vehicle group. ROS, reactive oxygen species; JPYQSX, Jianpi Yiqi Shexue.

in the control group $(\mathrm{P}<0.01)$; however, the mtDNA levels were comparable among the experiment groups after the administration of drugs (Figure 2, $\mathrm{P}>0.05$ ). The ATP levels in the colon were significantly decreased in the vehicle, prednisone, and low-dose JPQYSX groups (Figure 3, $\mathrm{P}<0.01$ ) and markedly increased in the high-dose JPQYSX group $(\mathrm{P}<0.01)$ when compared with the control group. After medication, the ATP levels in the colon were significantly increased; the high-dose JPQYSX group showed the highest ATP levels.

The protein and mRNA levels of SDHA in the spleen, heart, and brain tissues were markedly increased in the experiment groups compared to the control group (Figures 4,5); these levels were further decreased in the spleen after administration of prednisone and high-dose JPQYSX $(\mathrm{P}<0.05)$. Importantly, the protein and mRNA expressions of SDHA in the brain tissue were restored to normal levels after the administration of prednisone. Interestingly, the protein levels of SDHA were comparable in the liver, lung, and muscle tissues $(\mathrm{P}>0.05)$. However, the mRNA levels of SDHA were significantly increased in the vehicle group $(\mathrm{P}<0.05)$, while these levels were downregulated after administration of drugs $(\mathrm{P}<0.05)$.

The protein and mRNA levels of $\mathrm{ClpP}$ in the spleen, heart, and muscle tissues were significantly increased in the vehicle group compared to the control group $(\mathrm{P}<0.05)$. After drug administration, the $\mathrm{ClpP}$ levels in the spleen were decreased in the APS-induced mice $(\mathrm{P}<0.05)$. The levels of $\mathrm{ClpP}$ in the spleen were restored to the normal level in the low-dose JPQYSX and high-dose JPQYSX groups, while the levels of $\mathrm{ClpP}$ in muscle tissues were restored to normal in the prednisone and high-dose JPQYSX groups. Besides, prednisone and high-dose JPQYSX significantly decreased the ClpP levels in the heart, as compared to that in the vehicle group $(\mathrm{P}<0.05)$.

When compared to the control group, the vehicle 

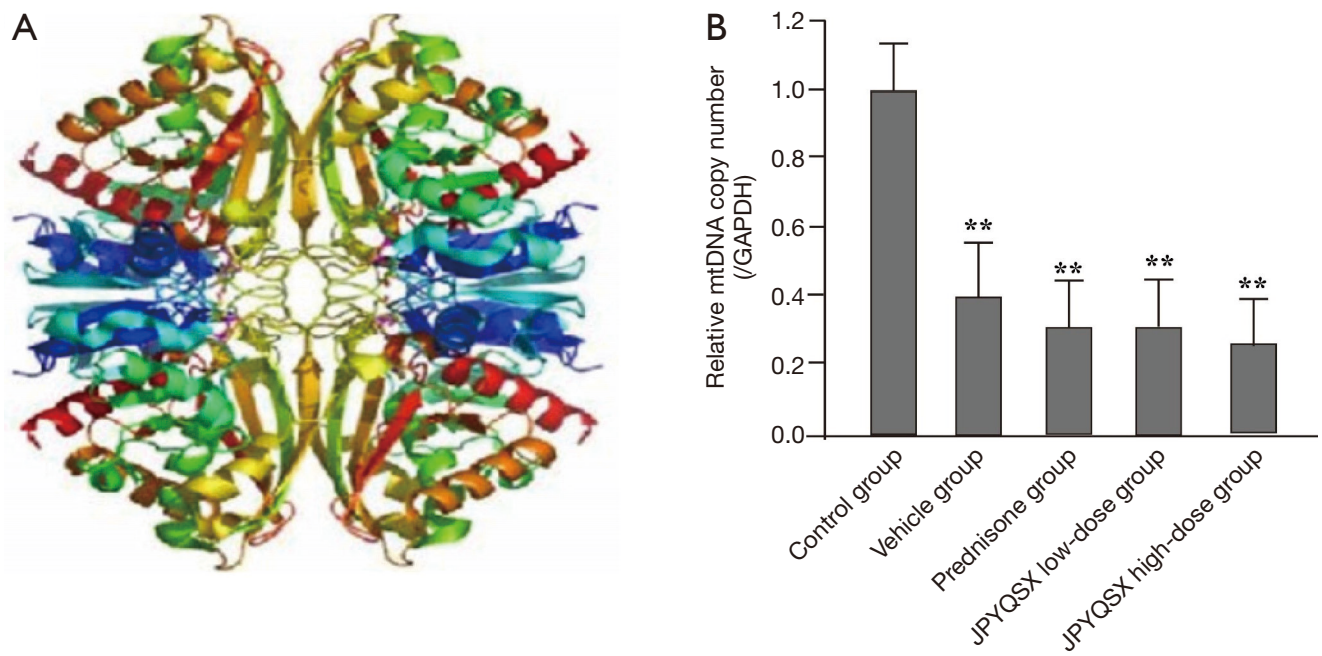

Figure 2 Relative mtDNA copy number. (A) 3D-structure of GAPDH gene. (B) Relative mtDNA copy number. The levels of mtDNA in the experiment group were significantly decreased as compared to that in the control group $\left.{ }^{* *} \mathrm{P}<0.01\right)$; however, the mtDNA levels in the various experiment groups were comparable after the administration of drugs $(\mathrm{P}>0.05)$. mtDNA, mitochondrial DNA; GAPDH, glyceraldehyde 3-phosphate dehydrogenase.
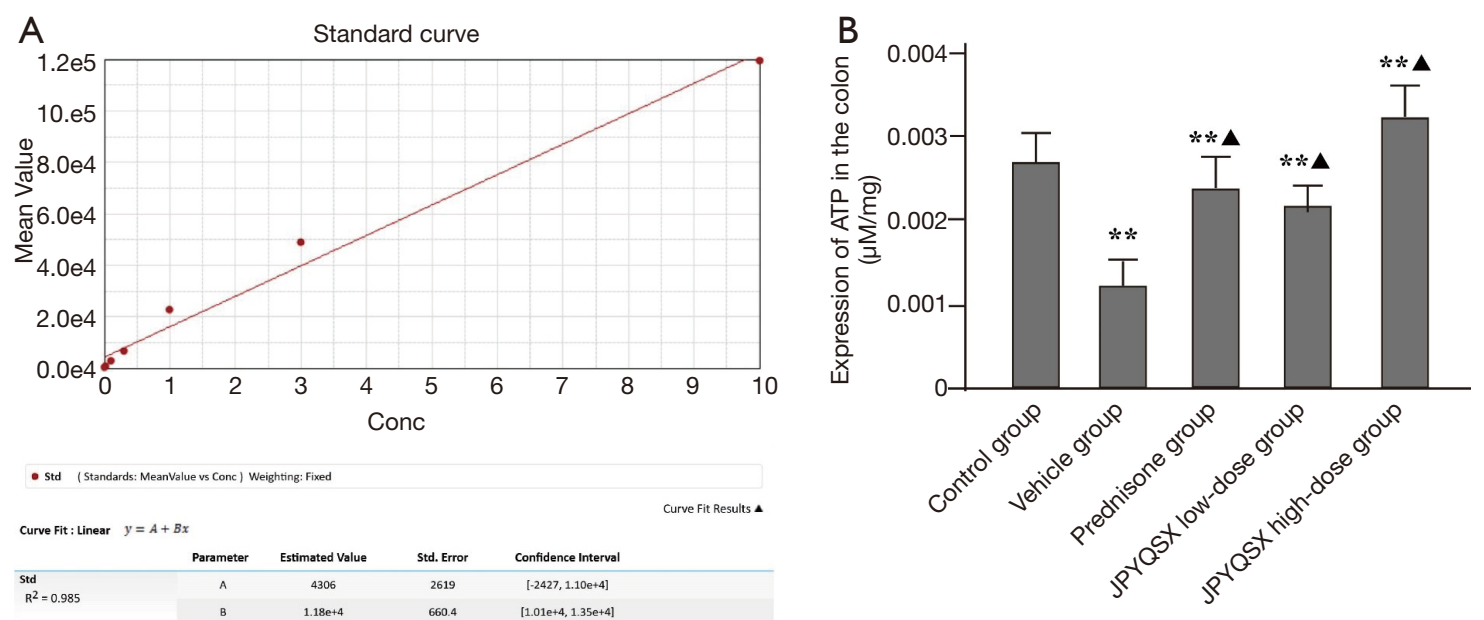

Figure 3 Expression level of ATP in colon tissues detected by ATP determination kit. (A) The standard curve of ATP in colon tissues of BALB/c mice. (B) Expression of ATP in the colon. Compared with the control group, the ATP levels in colon tissues were significantly decreased in the vehicle, prednisone, and low-dose JPYQSX groups ( $\left.{ }^{* *} \mathrm{P}<0.01\right)$, and markedly increased in the high-dose JPYQSX group $\left({ }^{\mathbf{A}} \mathrm{P}<0.01\right)$. ATP, adenosine triphosphate; JPYQSX, Jianpi Yiqi Shexue.

group showed a significant decrease in the protein and mRNA levels of LonP1 in the spleen and a significant increase in the heart and muscle tissues $(\mathrm{P}<0.05)$. After drug administration, the LonP1 levels in the spleen were increased in the prednisone group and were restored to a normal level $(\mathrm{P}<0.05)$. In addition, the LonP1 levels in the heart tissues were restored to the normal level after the administration of low-dose JPQYSX. Besides, LonP1 levels in muscles were also decreased in the prednisone and JPQYSX groups compared to the vehicle group $(\mathrm{P}<0.05)$.

\section{Discussion}

Bleeding is the most common symptom of ITP. However, 


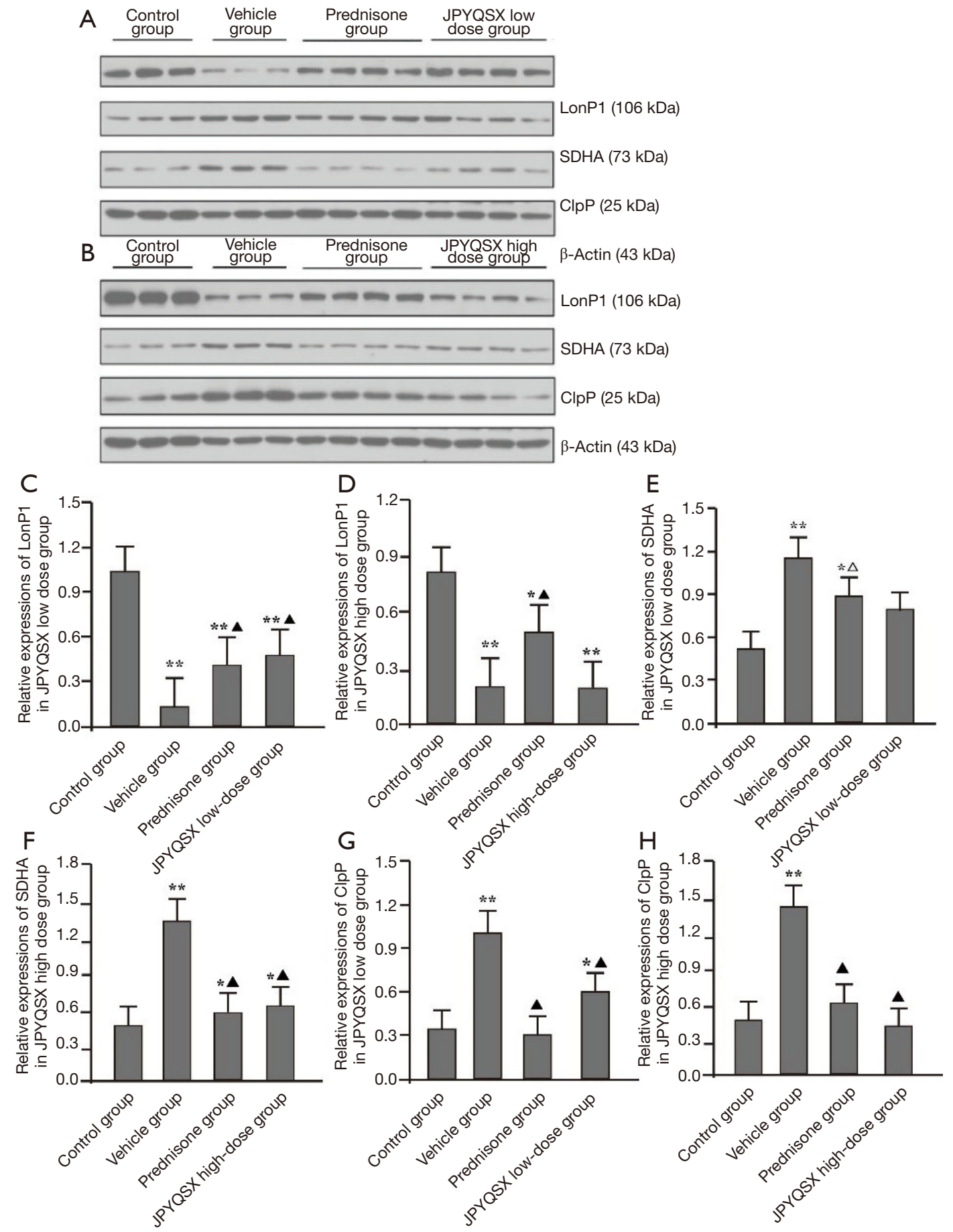

Figure 4 Results of western blot analysis showing the expression levels of SDHA, ClpP, and LonP1 in various groups. (A) Expressions of SDHA, ClpP, and LonP1 in the low-dose JPQYSX, control, vehicle, and prednisone groups. (B) Expressions of SDHA, ClpP, and LonP1 in the high-dose JPYQSX, control, vehicle, and prednisone groups. (C,D,E,F,G,H) Expression levels of SDHA, ClpP, and LonP1 presented as a ratio relative to the expression of GAPDH. ${ }^{*} \mathrm{P}<0.05$ versus control group; ${ }^{* *} \mathrm{P}<0.01$ versus control group; ${ }^{\triangle} \mathrm{P}<0.05$ versus vehicle group; ${ }^{\star} \mathrm{P}<0.01$ versus vehicle group. SDHA, succinate dehydrogenase complex flavoprotein subunit A; ClpP, caseinolytic mitochondrial matrix peptidase proteolytic subunit; LonP1, Lon peptidase 1; GAPDH, glyceraldehyde 3-phosphate dehydrogenase; JPYQSX, Jianpi Yiqi Shexue. 

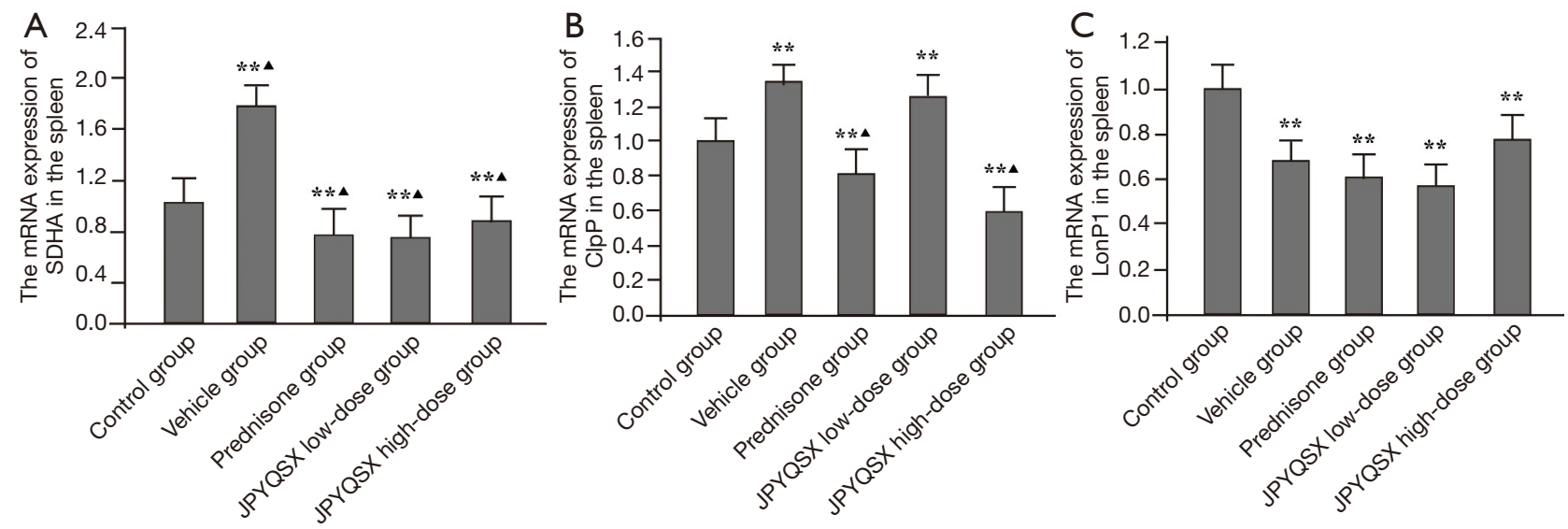

Figure 5 Results of qPCR showing mRNA expressions of SDHA, ClpP, and LonP1. (A) mRNA expression of SDHA; (B) mRNA expression of $\mathrm{ClpP}$; (C) mRNA expression of LonP1. ${ }^{* *} \mathrm{P}<0.01$ versus control group; ${ }^{\boldsymbol{\Delta}} \mathrm{P}<0.01$ versus vehicle group. qPCR, quantitative polymerase chain reaction; SDHA, succinate dehydrogenase complex flavoprotein subunit A; ClpP, caseinolytic mitochondrial matrix peptidase proteolytic subunit; LonP1, Lon peptidase 1 .

some patients with ITP may be asymptomatic and live with ITP for years without any episode of bleeding or visceral hemorrhage (1). Therefore, ameliorating the common symptoms of ITP (such as bleeding, fatigue, and shortness of breath) is a key imperative. In 2003, generic healthrelated quality of life (QoL) measures were developed for patients with ITP (13); these measures were aimed at optimizing the QoL of patients. Fatigue is the most common symptom in patients with ITP, irrespective of the presence or absence of bleeding. However, fatigue has only recently attracted the attention of clinicians and researchers. Although ITP-directed therapy can improve fatigue, there is a need to develop novel fatigue-directed strategies due to the limited effect of ITP-directed therapies (14). Studies have shown that the ROS level is markedly increased during physical exercise, while it is decreased under conditions of fatigue $(15,16)$. Moreover, oxidative stress resulting from mitochondrial dysfunction, the efficiency of mitochondrial ATP synthesis, and mitochondrial biogenesis are involved in the pathogenesis of fatigue (17-19). The main physiological functions of mitochondria include the generation of ATP, ROS, and synthesis and catabolism of metabolites. Abnormality in any of these processes can be termed mitochondrial dysfunction (20). In light of the above evidence, mitochondrial impairment or abnormalities of mitochondrial metabolism are important contributors to the development of fatigue in patients with ITP.

A clinical investigation concluded that most patients with ITP have a deficiency of qi or an impaired ability of the spleen to protect against hemorrhage; in addition, qisupplementing and yin-nourishing therapies were found to improve the TCM syndromes, including lassitude, dizziness, and palpitations $(8,21)$. The JPYQSX formula is traditionally used to treat primary ITP in the clinical practice of TCM (22). It has shown promising effects in elevating peripheral PLT counts; in addition, it has shown a prominent impact on antiplatelet antibody and immunological cytokines $(23,24)$. In the current study, we investigated the efficacy of JPYQSX on the blood picture and fatigue of mice with ITP from the perspective of mitochondrial activity. Administration of JPYQSX elevated the platelet counts to the same level as observed with prednisone; this indicates the potential efficacy of JPYQSX as a first-line drug. Administration of JPYQSX decreased the liver, lung, and spleen indices of mice and the effect was comparable to that of prednisone. Most patients with ITP ultimately develop hepatosplenomegaly (25), which is attributed to an autoimmune disorder, and may induce complications, such as abdominal distension and pain. Therefore, JPYQSX may be effective in inhibiting visceromegaly. The forced swimming test is a good method to evaluate physical power. Administration of JPYQSX doubled the swimming time of mice compared with the vehicle group; further, the performance exceeded that observed in the prednisone group. The data suggested that JPYQSX not only reduced the risk of bleeding by elevating the PLT counts, but also ameliorated fatigue of mice with ITP. These findings are consistent with those of a previous 
clinical study (22).

Rather than a homogenous disease, ITP is a systemic autoimmune disorder which is mainly attributed to immune deficiency and infection. However, in addition to the immune cells, various molecules are also involved in the pathogenesis of ITP. Among these, ROS is an important factor (26). Studies have demonstrated the activation of oxidative stress related gene pathways in patients with ITP; the ROS-modified proteins may trigger the production of autoantibodies $(27,28)$. In a recent study, increased mitochondrial activity and the consequent increase in oxidative phosphorylation were shown to severely impair the regeneration of hematopoietic stem cells, thereby resulting in the destruction of platelets (10). Moreover, antioxidant therapy was shown to ameliorate the bleeding tendency in patients with ITP (29). Importantly, the firstline therapy, methylprednisolone, can effectively reduce the oxidative stress in patients with ITP (30). Thus, oxidative activity might play a pivotal role in the process of ITP. In the present study, we found that JPYQSX elevated the levels of ROS, ATP, and mtDNA in mitochondria. This implies that JPYQSX may protect against platelet destruction and relieve fatigue via its effect on mitochondrial activity.

Functionally, $\mathrm{ClpP}$ is a mitochondrial matrix protease which plays a crucial role in mitochondrial protein turnover and bioenergetics activity (31). Overexpression of $\mathrm{ClpP}$ induces mitochondrial oxidative damage. In the present study, we demonstrated increased protein expression of ClpP in the ITP mice model, which was restored by JPYQSX. There is an involvement of SDHA in mitochondrial energy metabolism (32), and the mRNA expression of SDHA was reduced in the ITP mice model. However, it was elevated after treatment with JPYQSX and prednisone, which was consistent with the ATP level changes among the groups. The is a close relationship between LonP1and oxidative stress and aging, and its activity is essential for the maintenance of mitochondrial proteostasis $(33,34)$. Deficiency of LonP1 was shown to result in partial loss of mtDNA and suppression of mitochondrial translation; LonP1 upregulation was shown to induce the opposite changes in oxidative phosphorylation (35). In the present study, JPYQSX downregulated LonP1 expression to a normal level. However, the signaling pathways mediating the effects of JPYQSX are unknown at present. Previous studies revealed that MAPK1 signaling pathway was involved in the effects of Yiqi Shexue formula on primary ITP (36). Further studies are needed to clarify the signaling pathway underlying JPYQSX-mediated effects in ITP.

\section{Conclusions}

In summary, JPYQSX was found to be a potent formula for the treatment of ITP. It lowered the risk of hemorrhage and alleviated symptoms like fatigue. Mitochondrial related molecules, including ROS, ATP, and mtDNA were maladjusted in the ITP mice model. Treatment with JPYQSX remarkably restored their expression. Determination of SDHA, ClpP, and LonP1 further validated the impact of JPYQSX on mitochondrial activity. Further studies are required to comprehensively elucidate the underlying mechanism of the therapeutic effect of JPYQSX in ITP.

\section{Acknowledgments}

Funding: This research was supported by the National Science Foundation of China (81803904, 81703903).

\section{Footnote}

Reporting Checklist: The authors have completed the ARRIVE reporting checklist. Available at http://dx.doi. org/10.21037/apm-20-2153

Data Sharing Statement: Available at http://dx.doi. org/10.21037/apm-20-2153

Conflicts of Interest: All authors have completed the ICMJE uniform disclosure form (available at http://dx.doi. org/10.21037/apm-20-2153). The authors have no conflicts of interest to declare.

Ethical Statement: The authors are accountable for all aspects of the work in ensuring that questions related to the accuracy or integrity of any part of the work are appropriately investigated and resolved. All experiments were conducted in compliance with the Guidelines for the Care and Use of Laboratory Animals. Experimental protocols were approved by the Committee of Ethics of Animal Experimentation of the Air Force Medical University (No. XYLS2019069).

Open Access Statement: This is an Open Access article distributed in accordance with the Creative Commons Attribution-NonCommercial-NoDerivs 4.0 International License (CC BY-NC-ND 4.0), which permits the noncommercial replication and distribution of the article with 
the strict proviso that no changes or edits are made and the original work is properly cited (including links to both the formal publication through the relevant DOI and the license). See: https://creativecommons.org/licenses/by-nc-nd/4.0/.

\section{References}

1. Lambert MP, Gernsheimer TB, Clinical updates in adult immune thrombocytopenia. Blood 2017;129:2829-35.

2. Wang CY, Ma S, Bi SJ, et al. Enhancing autophagy protects platelets in immune thrombocytopenia patients. Ann Transl Med 2019;7:134.

3. Deane S, Teuber SS, Gershwin ME, The geoepidemiology of immune thrombocytopenic purpura. Autoimmun Rev 2010;9:A342-9.

4. Audia S, Mahevas M, Samson M, et al. Pathogenesis of immune thrombocytopenia. Autoimmun Rev 2017;16:620-32.

5. Lo E, Deane S, Diagnosis and classification of immunemediated thrombocytopenia. Autoimmun Rev 2014;13:577-83

6. Moulis G, Lapeyre-Mestre M, Montastruc JL, et al. Exposure to non-corticosteroid treatments in adult primary immune thrombocytopenia before the chronic phase in the era of thrombopoietin receptor agonists in France. A nationwide population-based study. Autoimmun Rev 2015;14:168-73.

7. Kojouri K, Vesely SK, Terrell DR, et al. Splenectomy for adult patients with idiopathic thrombocytopenic purpura: a systematic review to assess long-term platelet count responses, prediction of response, and surgical complications. Blood 2004;104:2623-34.

8. Liu WB, Li S, Yu XL, et al. Research Progress on Chinese Medicine Immunomodulatory Intervention for Chronic Primary Immune Thrombocytopenia: Targeting Cellular Immunity. Chin J Integr Med 2019;25:483-9.

9. Xie Y, Ge R, The Efficacy of Combination therapy of JianPi-Yi-Qi-She-Xue Prescription with RHTPO on Immune Thrombocytopenia Journal of Integrated Chinese and Western Medicine 2018;33:3696-9.

10. Vannini N, Campos V, Girotra M, et al. The NAD-Booster Nicotinamide Riboside Potently Stimulates Hematopoiesis through Increased Mitochondrial Clearance. Cell Stem Cell 2019;24:405-18.e7.

11. McDonald TP, A comparison of platelet production in mice made thrombocytopenic by hypoxia and by platelet specific antisera. Br J Haematol 1978;40:299-309.

12. Semple JW, Animal models of immune thrombocytopenia
(ITP). Ann Hematol 2010;89 Suppl 1:37-44.

13. Trotter P, Hill QA, Immune thrombocytopenia: improving quality of life and patient outcomes. Patient Relat Outcome Meas 2018;9:369-84.

14. Hill QA, Newland AC, Fatigue in immune thrombocytopenia. Br J Haematol 2015;170:141-9.

15. Cheng AJ, Yamada T, Rassier DE, et al. Reactive oxygen/ nitrogen species and contractile function in skeletal muscle during fatigue and recovery. J Physiol 2016;594:5149-60.

16. Westerblad H, Allen DG, Emerging roles of ROS/RNS in muscle function and fatigue. Antioxid Redox Signal 2011;15:2487-99.

17. McArdle A, McArdle F, Jackson MJ, et al. Investigation by polymerase chain reaction of enteroviral infection in patients with chronic fatigue syndrome. Clin Sci (Lond) 1996;90:295-300.

18. Moon Y, Balke JE, Madorma D, et al. Nitric Oxide Regulates Skeletal Muscle Fatigue, Fiber Type, Microtubule Organization, and Mitochondrial ATP Synthesis Efficiency Through cGMP-Dependent Mechanisms. Antioxid Redox Signal 2017;26:966-85.

19. Cho Y, Hazen BC, Gandra PG, et al. Perm1 enhances mitochondrial biogenesis, oxidative capacity, and fatigue resistance in adult skeletal muscle. FASEB J 2016;30:674-87.

20. Brand MD, Nicholls DG, Assessing mitochondrial dysfunction in cells. Biochem J 2011;435:297-312.

21. Zhou YH, Wei KM, He LY, et al. Multi-central clinical research into treating 80 cases of chronic thrombocytopenia with qi-supplementing and yinnourishing therapy and western medicine. J Tradit Chin Med 2011;31:277-81.

22. Wang J, Zhang L, Zhang Y, et al. The Clinical Efficacy of Jian-Pi-Yi-Qi-She-Xue Prescription on Immune Thrombocytopenia. Chinese Journal of Traditional Chinese Medicine 2018;12:5700-4.

23. He H, Zheng L, Xu Y, et al. The impact of Jian-Pi-Yi-SheXue Prexcription on perapheral blood picture in patients with ITP. Journal of Traditional Chinese Medicine, 2015;43:22-4.

24. Wu Y, Huang Z, Ma X, et al. The effect of Jian-PiYi-Qi-She-Xue Prescription on Antiplatelet Antibody and IL2, IL10, FOXP3 in Patients with Immune Thrombocytopenia. Journal of Fujian Medical University 2016;50:264-6.

25. Li P, Huang P, Yang Y, et al. Updated Understanding of Autoimmune Lymphoproliferative Syndrome (ALPS). Clin Rev Allergy Immunol 2016;50:55-63. 
26. Kamhieh-Milz J, Salama A, Oxidative stress is predominant in female but not in male patients with autoimmune thrombocytopenia. Oxid Med Cell Longev 2014;2014:720347.

27. Zhang B, Lo C, Shen L, et al. The role of vanin-1 and oxidative stress-related pathways in distinguishing acute and chronic pediatric ITP. Blood 2011;117:4569-79.

28. Zhang B, Zehnder JL, Oxidative stress and immune thrombocytopenia. Semin Hematol 2013;50:e1-4.

29. Erratum. Pediatr Blood Cancer 2015;62:1494.

30. Cura M, Koc A, Aksoy N, et al. Effect of short-term, high-dose methylprednisolone on oxidative stress in children with acute immune thrombocytopenia. Blood Res 2016;51:261-7.

31. Hu D, Sun X, Liao X, et al. Alpha-synuclein suppresses mitochondrial protease $\mathrm{ClpP}$ to trigger mitochondrial oxidative damage and neurotoxicity. Acta Neuropathol 2019;137:939-60.

32. Lavie J, De Belvalet H, Sonon S, et al. Ubiquitin-

Cite this article as: Zhang $\mathrm{Y}$, Wang $\mathrm{J}$, Gao J, Guo M, Chen H, Liu J, Li L, Liao J, Chen X, Lang H. Jianpi Yiqi Shexue ameliorates immune thrombocytopenia related fatigue by regulating mitochondrial function. Ann Palliat Med 2021;10(1):156-168. doi: 10.21037/apm-20-2153
Dependent Degradation of Mitochondrial Proteins

Regulates Energy Metabolism. Cell Rep 2018;23:2852-63.

33. Pomatto LC, Raynes R, Davies KJ, The peroxisomal Lon protease LonP2 in aging and disease: functions and comparisons with mitochondrial Lon protease LonP1. Biol Rev Camb Philos Soc 2017;92:739-53.

34. Zurita Rendón O, Shoubridge EA. LONP1 Is Required for Maturation of a Subset of Mitochondrial Proteins, and Its Loss Elicits an Integrated Stress Response. Mol Cell Biol 2018;38:e00412-17.

35. Gibellini L, Losi L, De Biasi S, et al. LonP1 Differently Modulates Mitochondrial Function and Bioenergetics of Primary Versus Metastatic Colon Cancer Cells. Front Oncol 2018;8:254.

36. Jiang Y, Liu N, Zhu S, et al. Elucidation of the Mechanisms and Molecular Targets of Yiqi Shexue Formula for Treatment of Primary Immune Thrombocytopenia Based on Network Pharmacology. Front Pharmacol 2019;10:1136. 